\title{
Carbon Nanofoam by Pulsed Electric Arc Discharges
}

\author{
David Saucedo-Jimenez, Isaac Medina-Sanchez, and Carlos Couder Castañeda
}

Centro de Desarrollo Aeroespacial, Instituto Politécnico Nacional, Belisario Dominguez 22, Centro, 06610 Ciudad de México, Mexico

Correspondence should be addressed to Carlos Couder Castañeda; ccouder@ipn.mx

Received 15 January 2018; Revised 23 April 2018; Accepted 8 May 2018; Published 23 August 2018

Academic Editor: Marco Cannas

Copyright ( 92018 David Saucedo-Jimenez et al. This is an open access article distributed under the Creative Commons Attribution License, which permits unrestricted use, distribution, and reproduction in any medium, provided the original work is properly cited.

\begin{abstract}
The aim of this article was to report the carbon nanofoam synthesis by a new method and a new catalytic mixture. Using the pulsed electric arc discharge method, carbon nanofoam was synthesized. The synthesis was carried out in a controlled atmosphere at 200 torr of hydrogen pressure. The pulsed electric arc discharge was established between two graphite electrodes with $22.8 \mathrm{kVA}$ of power and $150 \mathrm{~A}$ DC current; the cathode was relatively motionless and was made of a pure carbon rod of 6 mm diameter, and the spinner anode was a pure carbon disc spinning at $600 \mathrm{rpm}$; over the disc was an annular cavity where the new catalytic mixture of 93.84/2.56/1.43/0.69/1.48 of C/Ni/Fe/Co/S molar fraction was deposited in a geometrically fixed way by 8 catalytic mixture blocks and 8 empty spaces, and the discharge frequency was $80 \mathrm{~Hz}$. After the synthesis was made, the resulting products were deposited on the electrodes, proving that our method can synthesize different carbon nanostructures easily and at low cost.
\end{abstract}

\section{Introduction}

Synthesized materials include many further distinct forms of carbon such as fullerenes, multiwalled carbon nanotubes, and single-walled carbon nanotubes, which are based on a mixture of $\mathrm{sp}^{2}$ and $\mathrm{sp}^{3}$ hybridized carbon atoms. The structural phase space of carbon between graphite-like and diamond-like hybridization states now has many occupants.

There is even more variety in the electronic properties of various carbon allotropes, which range from superconductivity to ferromagnetism and tunable electrical conductivity [1].

Nanofoams are of considerable current interest due to their unique structure, which lies between two and three dimensions, allowing for many new types of materials with promising new functions for future technologies.

Since many material functions rely on molecule surface interactions and low-dimensional properties, materials with large surface areas and a quantum-confined nanoscale nature are of particular interest. Such conditions are provided by porous materials with nanometer dimensions [2].

Nanofoams of different materials have been used for specific applications, varying from medicinal applications by using nickel nanofoam [3] to semiconductors, insulators, and acoustic-optic materials based on silicon $[4,5,6]$ or to simply characterize other nanofoam structures such as gold and titanium nanofoams using hydrogen nanofoams [7].

The possibility to produce foams with controlled and reproducible mean density, area, and thickness, with a satisfactory adhesion on a solid substrate, might be significant to achieve desired material properties and/or for specific applications. Carbon represents a particularly suitable choice as foam constituent since, being a light element, it makes it easier to reach low densities, and because it can in principle lead to a monoelemental film due to its volatile oxides [8].

Pulsed laser deposition (PLD) has been proven as one of the most popular ways of synthesizing carbon nanofoams since it creates highly uniform samples, good adhesion to the substrate, and high porosity, with the possibility of reducing the density range by varying the forming conditions.

While many researchers focus on improving the uniformity and reducing the density range, few have proposed new methods for the formation of carbon nanofoams. Such is the case of Mitchell et al. [9], which proposal is to form high-quality carbon nanofoam via low-temperature 
hydrothermal carbonization of sucrose. Their fundamental idea for this was to develop an environmentally friendly method, without the use of any chemical. Using this technique, the foams tend to be composed of micrometer-sized spheres of predominantly $\mathrm{sp}^{2}$ carbon that forms a threedimensional open scaffold. These so-called micropearls are usually detected as individual units which are weakly connected, forming the foam structure. Their interaction is not strong enough for coalescence to occur. The hydrothermal process allows for the variation of growth parameters, which may lead to further foam morphologies. The study of the parameter-morphology relationship can help to better understand the hydrothermal carbonization process, and in addition, to tune the growth toward particular material structures.

Some methods for improving the formation of carbon nanofoam include the one mentioned by Zel'dovich et al. [10] that has been used since then with many variations of gas density or laser power, where it is stated that the presence of a gas atmosphere during the PLD process may have two different objectives: the use of a chemically reactive gas is necessary either to correct incongruent ablation or to introduce in the deposited film atomic species that are lacking in the target material.

Typically, the pressure of the ambient gas is kept low to preserve the energy of the atomic species impinging onto the surface, thus allowing compact, uniform films to grow. In another scenario, inert gases at high pressure are mostly used. When laser-generated plasma becomes spatially confined, most of its initial kinetic energy is converted into thermal energy, leading to a rise of the temperature up to several thousands of Kelvin degrees. Under such conditions, mutual aggregation of plasma constituents is favored, thus opening the way to the synthesis of nanoparticles during plasma propagation and possibly to the growth of nanostructured materials by PLD [11].

Also, Filipescu et al. [12] have proposed radiofrequencyassisted pulsed laser deposition for the creation of $\mathrm{WO}_{x}$ (tungsten oxide) films. This favors the formation of nanostructured agglomerates that uniformly cover the substrate surface for this particular sample.

In this paper, a new method for synthesizing carbon nanofoam is proposed by using pulsed arch electric discharges in a low-frequency controlled scenario and a new proposal for the catalytic mixture. This is a modification of previous reported analysis that has been proven to work under the circumstances described throughout this work.

\section{Experimental Section}

The synthesis of carbon nanofoam by pulsed electric arc discharge with spinner anode was located inside the chamber developed by Saucedo-Jimenez et al. [13]. The chamber is made of 304s stainless steel, which can be easily manipulated to perform the experimental setup, and the body can be removed from the base, likewise, the upper part of the body has a cover that can be removed (Figure 1(a)). At the side of the chamber body, there is a boron-silicate observation window transversely to access and do any possible adjustment not contemplated during the experimental setup and to make visual contact during the development of the experiment; this window can also be removed. The chamber temperature is controlled by a cold-water circulation cooling system. In order to perform the pulsed and periodic electric arc discharge, two electrodes with a potential difference between them were considered; these electrodes have a $3 \mathrm{~mm}$ minimum gap to generate the electric arch discharge; however, this setup is made in such a way that this gap varies in time, so that the electric arc cannot be established continuously. Figure 1(b) shows the configuration of the components inside the chamber.

The proximity and separation of the electrodes have been made by a mechanical means, by using a discontinuous circular anode, formed by catalytic mixture blocks and empty blocks. The cathode was located in a relatively motionless position, thus maintaining the specified gap, and can only move a few millimeters in a vertical way. The anode is a spinning carbon disc of $10 \mathrm{~cm}$ diameter with a constant circular motion, and over this disc, there is a channel of $3.5 \mathrm{~cm}$ of radius, $5 \mathrm{~mm}$ depth, and $1.5 \mathrm{~cm}$ width, as shown in Figure 2(a). When a catalytic mixture block approaches the cathode, the gap reduces and the discharge is established. At a subsequent time, when an empty sector of the anode reaches the cathode, the gap between the electrodes increased and the arc discharge was suspended. With this mechanism, a pulsed and periodic electric arc discharge is generated. The anode motion was constant considering its geometrical configuration, and a periodical catalytic mixture deposition was done; hence, the pulsed discharge was periodical too. The cathode is a pure sharpened graphite rod of $6 \mathrm{~mm}$ diameter to favor the emission of electrons by the tip effect, as shown in Figure 2(b).

Within the channel, a catalytic mixture is deposited, forming compacted catalytic mixture blocks distributed in a special geometric design, establishing sectors with catalytic mixture (teeth) and sectors without this; the arrangement of this mixture was forming 16 sectors of the same dimensions, thus forming 8 sectors of catalytic mixture and 8 sectors without mixing.

The constant angular velocity is set at $600 \mathrm{rpm}$, so the discharge time is of $6 \mathrm{~ms}$ approximately, which is a reasonable time to allow a short growth of the nanotube before an abrupt change in the environment, where it is immersed as reported by Puretzky et al. [14].

The direction of the rotation was set counterclockwise; this rotation generates a drag of the jet plasma, and this effect increases the plasma volume, thus having a greater space for the nucleation and growth of the nanostructures. This growth is achieved due to the angle of the cathode that is located relatively normal to the anode and is of about $60^{\circ}$. The catalytic mixture employed was as follows: $93.84 / 2.56 / 1.43 / 0.69 / 1.48$ of $\mathrm{C} / \mathrm{Ni} / \mathrm{Fe} / \mathrm{Co} / \mathrm{S}$ in molar fractions. This catalytic mixture is a modification to that used by Liu et al. [15] and Tibbetts et al. [16], by using this, it is intended to induce topological defects as pentagonal rings by increasing the sulfur concentration to a double proportion; also, this mixture adds the sulfur in its elemental stage, while in the original, the sulfur is added as FeS. 


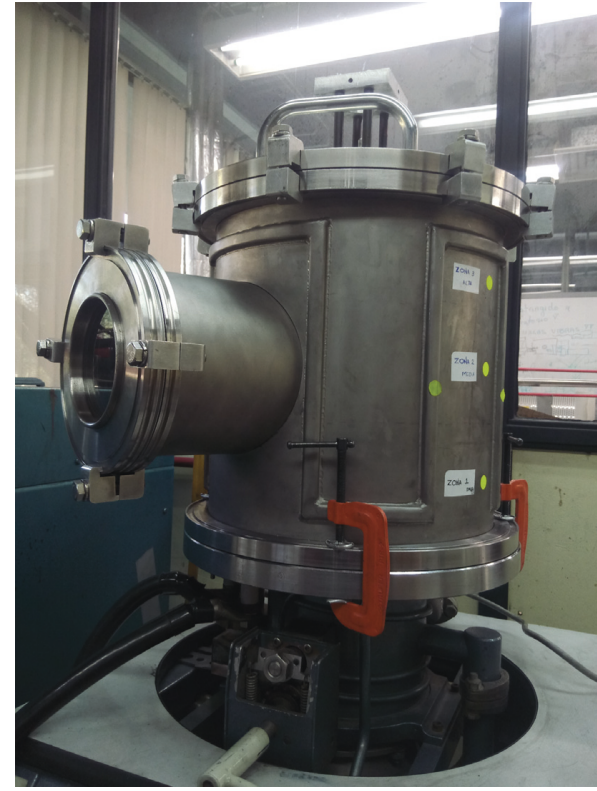

(a)

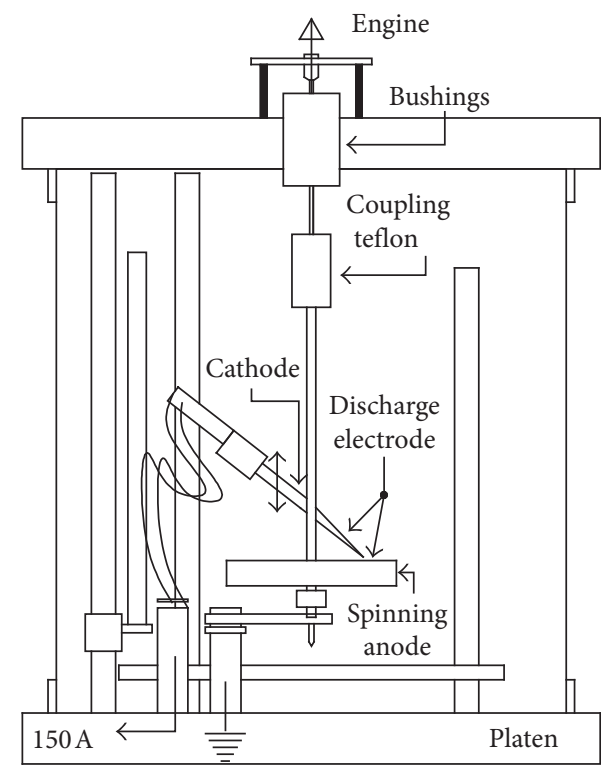

(b)

Figure 1: Carbon nanostructures growth chamber. (a) Growth chamber. (b) Growth chamber CAD design.

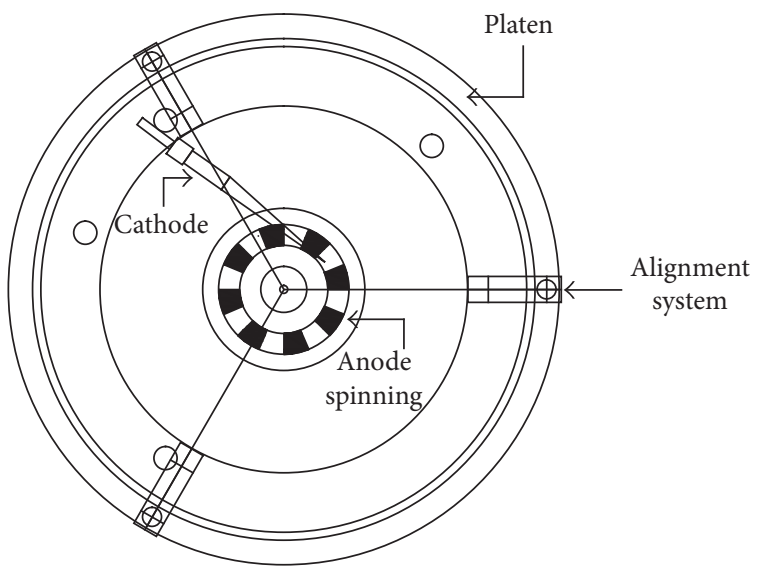

(a)

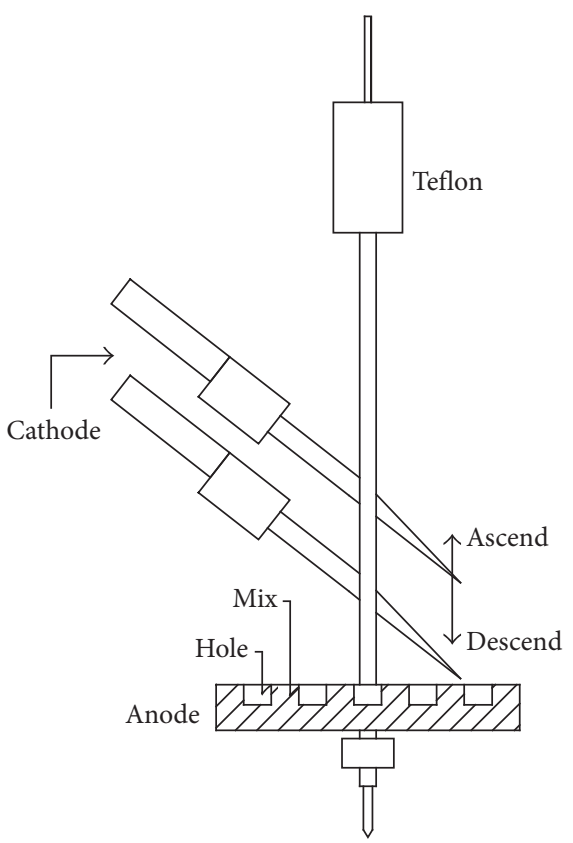

(b)

Figure 2: Carbon nanostructures growth chamber interior design. (a) Growth chamber top view. (b) Ascendant and descendant moving tip mechanism.

\section{Results and Discussions}

The first result after the experiment showed no deposition of nanotubes in spiderweb form; as a result, there was no growth of single wall or few wall carbon nanotubes, possibly due to excess sulfur concentration; nevertheless, carbon nanostructures were deposited on the walls of the chamber and on the surface of the electrodes. The material deposited on the walls of the chamber was characterized by scanning electron microscopy and was found mostly as amorphous carbon. The material deposited in the electrodes was characterized by the same microscopy, and a different material was found; this material showed very porous nanostructures, similar to fractals. This material was 
characterized by scanning and transmission electron microscopy as well as by Raman spectroscopy.

3.1. Scanning Electron Microscopy. The samples collected at the electrodes (cathode and anode) were characterized by scanning electron microscopy using the microscope mark FEI model Nova 200 NanoLab dual beam, focused ion beam (FIB), finding similar structures in both electrodes with the difference that the structures deposited in the cathode are free of amorphous carbon and catalyst particles than those deposited in the anode.

Figures 3 and 4 show the synthesized product that was observed and deposited on the surface of the anode. Structures highly contaminated by a large amount of amorphous carbon were found to be $35.66 \%$, while the number of carbon nanofoam was $11.94 \%$, and the proportion of catalyst particles was $52.4 \%$. Figure $3(3000 x$ magnification) shows large rope formations with dimensions around 1 micron in width, and the ropes observed could be formed by carbon nanotubes. By the scanning electron microscopy characterization, we observed the rope surface, finding what could possibly be carbon nanotubes with diameters of about $30 \mathrm{~nm}$. Figure 4 (6000x magnification) shows formation consisting of carbon nanotubes and catalyst particles, and the nanotube diameters are approximately $35 \mathrm{~nm}$, while the particle diameters are on average $380 \mathrm{~nm}$.

The morphology of the synthesized and deposited nanostructures on the surface of the cathode is shown in Figures 5 and 6; those nanostructures are similar to the ones shown in Figures 3 and 4, with the difference that they are not excessively contaminated by amorphous carbon, with an amorphous carbon proportion of $15.46 \%$, while the proportion of nanofoam carbon is of $81.82 \%$, and the proportion of catalyst particles is $2.71 \%$. The structure of the sample resembles a fractal, a typical shape of the carbon nanofoam; the dimensions of the nanotube-like structures that shape the carbon nanofoam are $2.7 \mathrm{~nm}$ in diameter and approximately $100 \mathrm{~nm}$ in length, before breaking translation symmetry and changing its direction.

The proportion of amorphous carbon was calculated by making a very fine mesh on the micrograph.

3.2. Raman Spectroscopy. Resonance Raman spectra from samples collected at the electrodes (cathode and anode) are acquired using the confocal micro-Raman spectrometer Horiba Jobin Yvon (LabRam HR800), with CCD detector, power resolution $1024 \times 256$ pixels, variable spot size in range from 0.86 to $3.1 \mu \mathrm{m}$, optimized spectral range of 200-1100 nm (100-4500 $\left.\mathrm{cm}^{-1}\right)$, with laser lines of $532.07 \mathrm{~nm}$ $(2.34 \mathrm{eV}$ green with power $43.4 \mathrm{~mW}), 632.79 \mathrm{~nm}(1.97 \mathrm{eV}$ red with power $6.3 \mathrm{~mW})$, and $784.12 \mathrm{~nm}(1.48 \mathrm{eV}$ near infrared with power of $56.7 \mathrm{~mW}$ ).

The spectrometer uses a confocal microscope (Olympus Bx41) with three objective lens of $10 x, 50 x$ and $100 x$ magnification. The spectrometer have gratings of $300 \mathrm{gr} / \mathrm{mm}$, $600 \mathrm{gr} / \mathrm{mm}, 950 \mathrm{gr} / \mathrm{mm}$, and $1800 \mathrm{gr} / \mathrm{mm}$. For $100 \mathrm{x}$

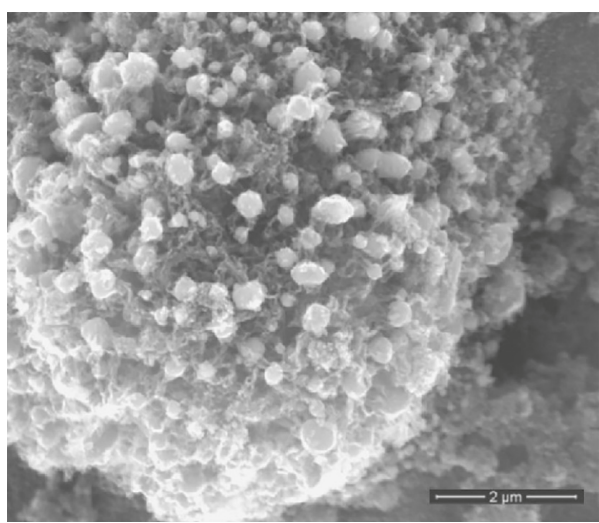

Figure 3: Anode magnification, 3000x.

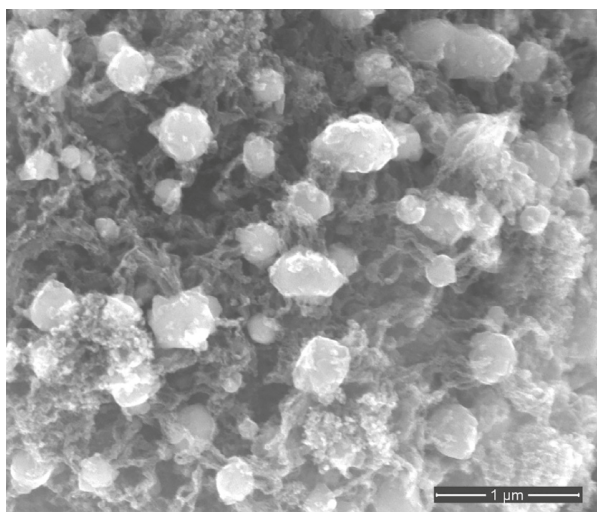

Figure 4: Cathode magnification, 6000x.

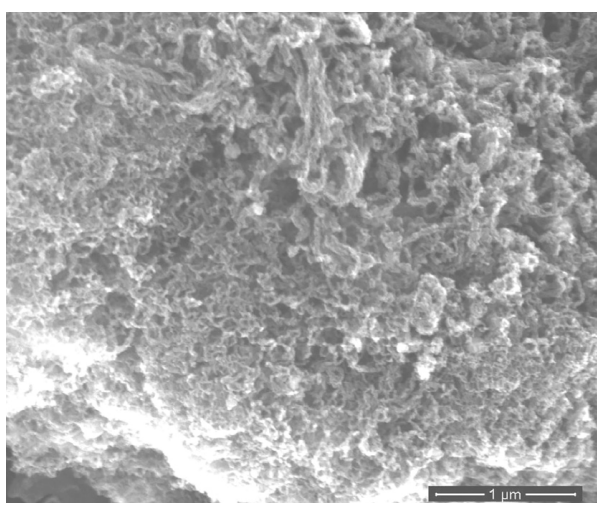

FIgURE 5: Cathode magnification, 6000x.

magnification in high resolution mode, the spectral resolution is $0.3 \mathrm{~cm}^{-1}$. The process of measuring the Raman spectra for carbon nanofoam is made on a soda lime silicate safety glass substrate.

In the scanning electron microscopy, it is observed that this carbon nanofoam is conformed by structures similar to carbon nanotubes; therefore, the Raman spectroscopy characterization is proposed, to analyze the spectrum with the theory developed for carbon nanotubes [17, 18], and 


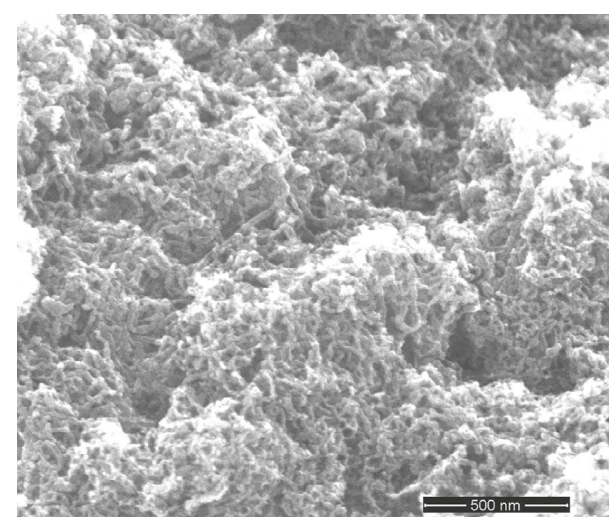

Figure 6: Cathode magnification, 12000x.

a possible interpretation to the signals obtained by this technique is stated.

By means of this characterization, it is determined that the structure of the carbon nanofoam is not formed by carbon nanotubes of few walls since no response was obtained in the region RBM $50-400 \mathrm{~cm}^{-1}$, characteristic of radial breathing mode vibrations of the carbon atoms; however, a response is obtained in the region of $1200-1700 \mathrm{~cm}^{-1}$, corresponding to the tangential vibrations of the carbon atoms that have the nanotubes. In this same region, there is the response of disorder band corresponding to heteroatoms, vacancies, heptagon-pentagon pairs, or even the presence of impurities, and so on. Considering that the possible carbon nanotubes in the sample are multiwalled carbon nanotubes with lengths of $100 \mathrm{~nm}$ approximately, before breaking the translation symmetry, it is clear that the response in the region $1450 \mathrm{~cm}^{-1}$ corresponds to disorderinduced $\mathrm{D}$ band due to amorphous carbon and heptagonpentagon pairs present in the sample, as was mentioned in the scanning electron microscopy. Figures 7-12 give a general view of the Raman spectra from samples of carbon nanofoam. The signal shows the Raman features corresponding to tangential modes ( $\mathrm{G}$ band) and disorderinduced $\mathrm{D}$ band. The $\mathrm{D}$-band intensity usually is 100 times smaller than that of the G band; large D-band peaks compared with the G-peak intensity usually indicate the presence of amorphous carbon [19]. For the Raman spectra analysis, usually there are two characteristics that differentiate the $\mathrm{D}$ band: defects in the structure carbon nanotubes and amorphous carbon. The Raman spectra from carbon nanotubes are composed of a broad peak upon which is superimposed a sharper peak; the broad feature refers to amorphous carbon, and the sharper feature refers to the carbon nanotube defects [20].

The fit parameters of the experimental data obtained from the Raman characterization are shown in Tables 1-6.

3.2.1. Fit Parameters for Raman Spectra Data in Anode. Table 1 shows the adjustment of the $G$ region or tangential vibrations; the first two signals correspond to defects in the nanotubes; of these, the narrowest signal, $1338.13 \mathrm{~cm}^{-1}$, corresponds to bent nanotubes that in this case correspond

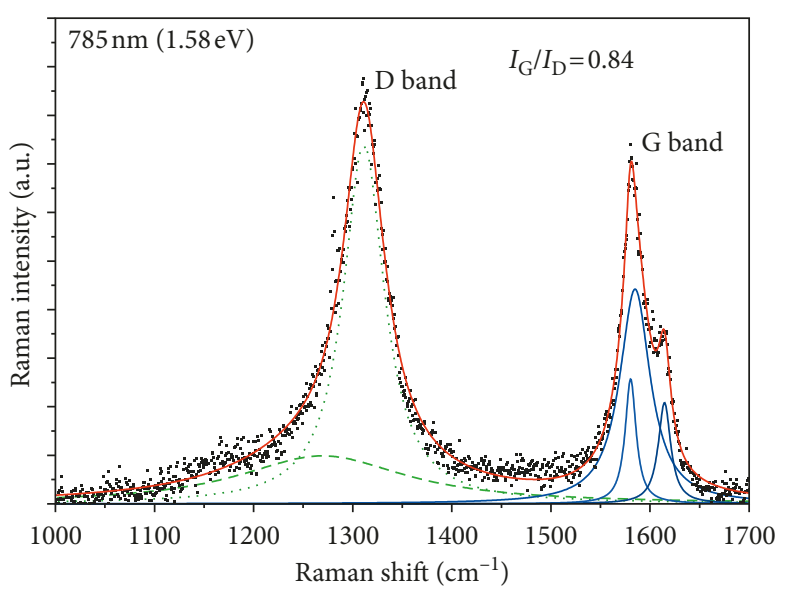

FIgUre 7: Cathode Raman spectra, $1.58 \mathrm{eV}$.

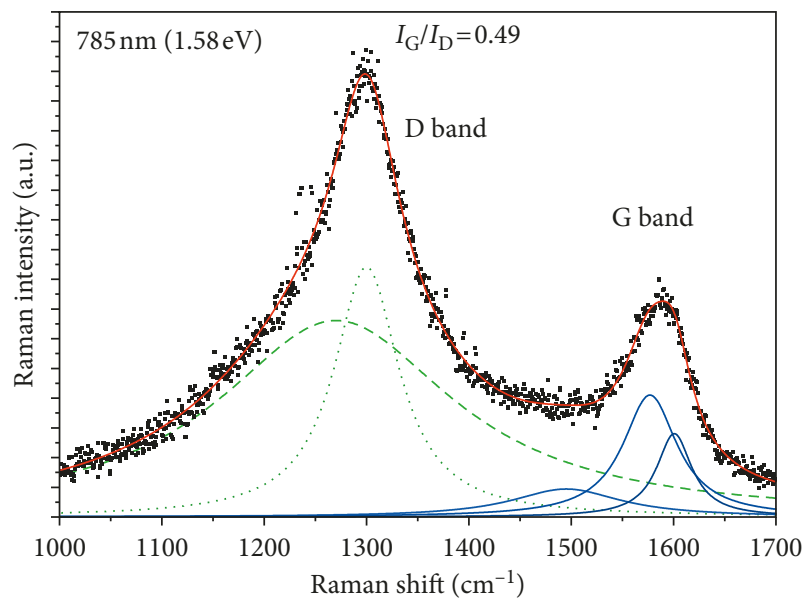

Figure 8: Anode Raman spectra, $1.58 \mathrm{eV}$.

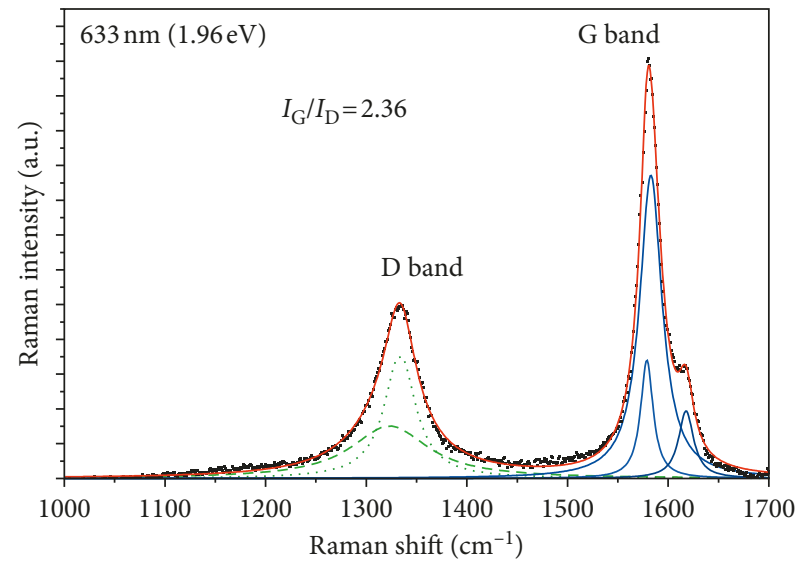

Figure 9: Cathode Raman spectra, $1.96 \mathrm{eV}$.

to carbon nanofoam. The widest signal, $1346.26 \mathrm{~cm}^{-1}$, corresponds to amorphous carbon. The last three signals correspond to tangential vibrations of the carbon atoms, the signal in $1549.20 \mathrm{~cm}^{-1}$ corresponds to the circumferential 


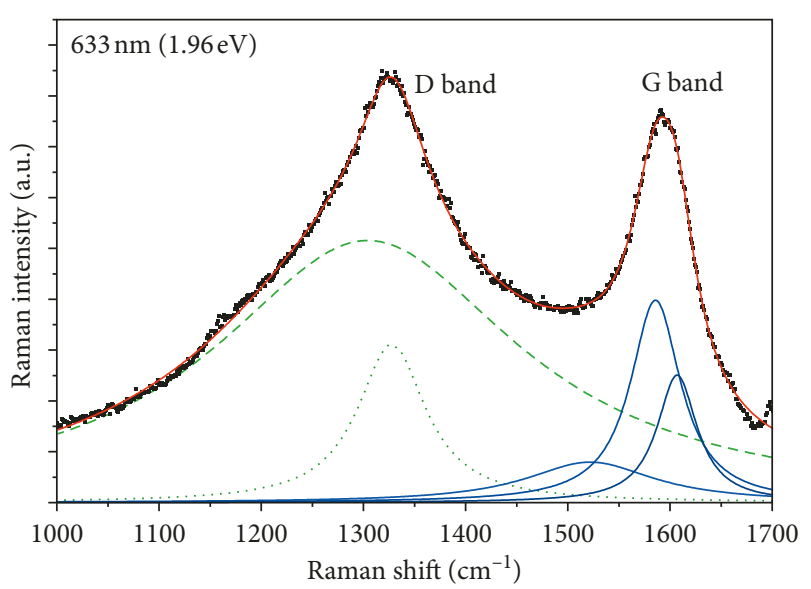

Figure 10: Anode Raman spectra, $1.96 \mathrm{eV}$.

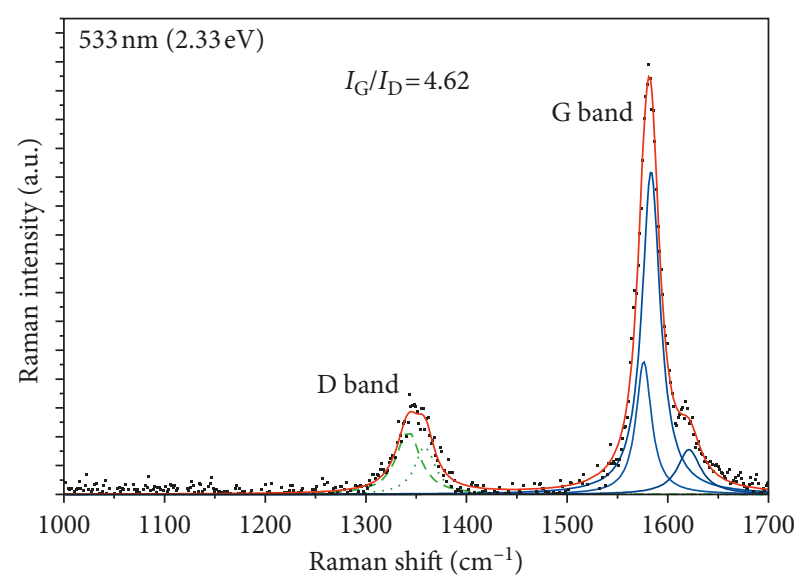

FIgURe 11: Cathode Raman spectra, $2.33 \mathrm{eV}$.

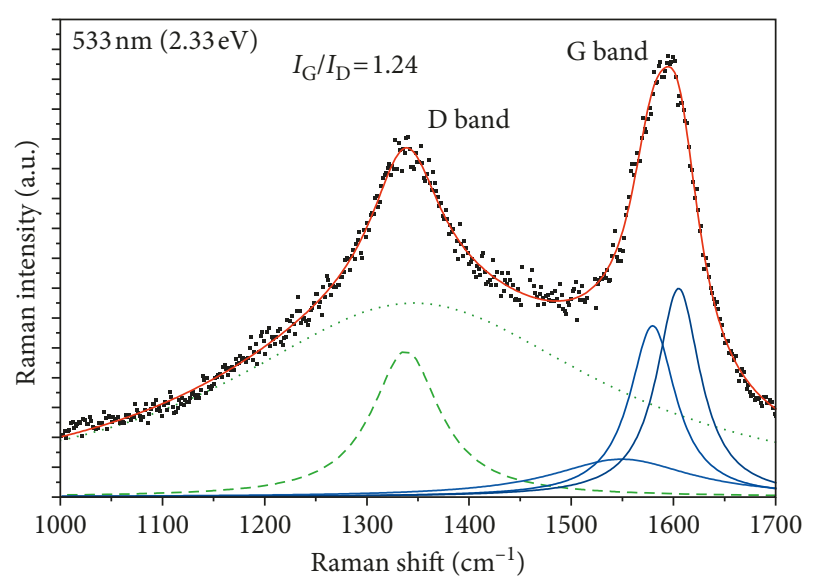

Figure 12: Anode Raman spectra, $2.33 \mathrm{eV}$.

vibration, the second to the vibration in $1579.64 \mathrm{~cm}^{-1}$ corresponds to the carbon materials with $\mathrm{sp}^{2}$ hybridization, and the last signal in $1605.03 \mathrm{~cm}^{-1}$ corresponds to the vibration in the direction parallel to the axis tube.

Table 2 shows the adjustment of the $\mathrm{G}$ region or tangential vibrations; the first two signals correspond to defects in the nanotubes; of these, the narrowest signal, $1327.73 \mathrm{~cm}^{-1}$, corresponds to bent nanotubes that in this case correspond to carbon nanofoam. The widest signal, $1304.29 \mathrm{~cm}^{-1}$, corresponds to amorphous carbon. The last three signals correspond to tangential vibrations of the carbon atoms, the signal in $1521.89 \mathrm{~cm}^{-1}$ corresponds to the circumferential vibration, the second to the vibration in $1585.84 \mathrm{~cm}^{-1}$ corresponds to the carbon materials with $\mathrm{sp}^{2}$ hybridization, and the last signal in $1606.99 \mathrm{~cm}^{-1}$ corresponds to the vibration in the direction parallel to the axis tube.

Table 3 shows the adjustment of the $G$ region or tangential vibrations; the first two signals correspond to defects in the nanotubes; of these, the narrowest signal, $1299.98 \mathrm{~cm}^{-1}$, corresponds to bent nanotubes that in this case correspond to carbon nanofoam. The widest signal, $1270.23 \mathrm{~cm}^{-1}$, corresponds to amorphous carbon. The last three signals correspond to tangential vibrations of the carbon atoms, the signal in $1495.12 \mathrm{~cm}^{-1}$ corresponds to the circumferential vibration, the second to the vibration in $1576.89 \mathrm{~cm}^{-1}$ corresponds to the carbon materials with $\mathrm{sp}^{2}$ hybridization, and the last signal in $1600.65 \mathrm{~cm}^{-1}$ corresponds to the vibration in the direction parallel to the axis tube.

Table 7 shows the proportions of amorphous carbon, and defects in nanostructures are summarized. Typical characteristics of the Raman spectra of carbon SP2 bonds are the G and $\mathrm{D}$ bands. The $\mathrm{G}$ band of tangential stretching is located approximately on $1580 \mathrm{~cm}^{-1}$ and corresponds to the carbon atoms vibrations in the tangential direction, that is, over the plane that defines the hexagons formed by the carbon atoms. The $\mathrm{D}$ band of induced disorder, located between 1250 and $1450 \mathrm{~cm}^{-1}$, describes the presence of substitutional heteroatoms, vacancies, grain boundaries, topological defects by finite size effects, and the presence of other carbon materials (amorphous carbon covering the nanotubes), among other defects. The intensities of these bands are known as IG and ID; the defect index (IG/ID) is useful for an approximation that indicates statistically the concentration of topological defects, rehybridization defects, incomplete links, and the presence of amorphous carbon.

3.2.2. Fit Parameters for Raman Spectra Data in Cathode. Table 4 shows the adjustment of the $G$ region or tangential vibrations; the first two signals correspond to defects in the nanotubes; of these, the narrowest signal, $1358.76 \mathrm{~cm}^{-1}$, corresponds to bent nanotubes that in this case correspond to carbon nanofoam. The widest signal, $1342.01 \mathrm{~cm}^{-1}$, corresponds to amorphous carbon. The last three signals correspond to tangential vibrations of the carbon atoms, the signal in $1576.10 \mathrm{~cm}^{-1}$ corresponds to the circumferential vibration, the second to the vibration in $1583.39 \mathrm{~cm}^{-1}$ corresponds to the carbon materials with $\mathrm{sp}^{2}$ hybridization, and the last signal in $1621.05 \mathrm{~cm}^{-1}$ corresponds to the vibration in the direction parallel to the axis tube.

Table 5 shows the adjustment of the G region or tangential vibrations; the first two signals correspond to defects in the nanotubes; of these, the narrowest signal, $1323.10 \mathrm{~cm}^{-1}$, corresponds to bent nanotubes that in this 
TABLE 1: Fitted parameters anode energy, $2.33 \mathrm{eV}(533 \mathrm{~nm})$.

\begin{tabular}{|c|c|c|c|c|c|c|}
\hline Peak & Type & Amplitude (a.u.) & Center $\left(\mathrm{cm}^{-1}\right)$ & FWHM $\left(\mathrm{cm}^{-1}\right)$ & $\%$ area & Interpretation \\
\hline 1 & Lorentz amp & 97.95 & 1338.13 & 80.60 & 12.22 & Nanotube defects \\
\hline 2 & Lorentz amp & 129.97 & 1346.26 & 443.91 & 61.67 & Amorphous carbon \\
\hline 3 & Lorentz amp & 25.38 & 1549.20 & 166.85 & 5.60 & $\mathrm{G}-$ \\
\hline 4 & Lorentz amp & 114.78 & 1579.64 & 54.88 & 9.62 & G0 \\
\hline 5 & Lorentz amp & 139.76 & 1605.03 & 51.73 & 10.90 & G+ \\
\hline
\end{tabular}

Laser characterization energy $=2.33 \mathrm{eV}$. The coefficient of determination $r^{2}=0.99$.

TABLE 2: Fitted parameters anode energy $1.96 \mathrm{eV}(633 \mathrm{~nm})$.

\begin{tabular}{lcccccc}
\hline Peak & Type & Amplitude (a.u.) & Center $\left(\mathrm{cm}^{-1}\right)$ & FWHM $\left(\mathrm{cm}^{-1}\right)$ & \% area & Interpretation \\
\hline 1 & Lorentz amp & 1030.99 & 1304.29 & 362.07 & 80.96 & Amorphous carbon \\
2 & Lorentz amp & 623.54 & 1327.73 & 153.65 & 5.17 & Nanotube defects \\
3 & Lorentz amp & 159.26 & 1521.89 & 59.84 & 11.02 & G- \\
4 & Lorentz amp & 796.23 & 1585.84 & 47.79 & 5.57 & G0 \\
5 & Lorentz amp & 501.17 & 1606.99 & $\mathrm{G}+$ & \\
\hline
\end{tabular}

Laser characterization energy $=1.96 \mathrm{eV}$. The coefficient of determination $r^{2}=0.99$.

TABLE 3: Fitted parameters anode energy $1.58 \mathrm{eV}(783 \mathrm{~nm})$.

\begin{tabular}{|c|c|c|c|c|c|c|}
\hline Peak & Type & Amplitude (a.u.) & Center $\left(\mathrm{cm}^{-1}\right)$ & FWHM $\left(\mathrm{cm}^{-1}\right)$ & $\%$ area & Interpretation \\
\hline 1 & Lorentz amp & 132.23 & 1270.23 & 280.15 & 56.72 & Amorphous carbon \\
\hline 2 & Lorentz amp & 168.23 & 1299.98 & 77.28 & 24.73 & Nanotube defects \\
\hline 3 & Lorentz amp & 18.83 & 1495.12 & 130.65 & 4.33 & $\mathrm{G}-$ \\
\hline 4 & Lorentz amp & 82.05 & 1576.89 & 64.56 & 9.78 & G0 \\
\hline 5 & Lorentz amp & 56.05 & 1600.65 & 41.97 & 4.45 & $\mathrm{G}+$ \\
\hline
\end{tabular}

Laser characterization energy $=1.58 \mathrm{eV}$. The coefficient of determination, $r^{2}=0.99$.

TABLe 4: Fitted parameters cathode energy $2.33 \mathrm{eV}(533 \mathrm{~nm})$.

\begin{tabular}{|c|c|c|c|c|c|c|}
\hline Peak & Type & Amplitude (a.u.) & Center $\left(\mathrm{cm}^{-1}\right)$ & FWHM $\left(\mathrm{cm}^{-1}\right)$ & $\%$ area & Interpretation \\
\hline 1 & Lorentz amp & 22.15 & 1342.01 & 28.31 & 13.27 & Amorphous carbon \\
\hline 2 & Lorentz amp & 17.00 & 1358.76 & 23.44 & 8.46 & Nanotube defects \\
\hline 3 & Lorentz amp & 46.02 & 1576.10 & 18.53 & 17.87 & $\mathrm{G}-$ \\
\hline 4 & Lorentz amp & 112.04 & 1583.39 & 21.93 & 51.44 & G0 \\
\hline 5 & Lorentz amp & 15.56 & 1621.05 & 27.94 & 8.85 & $\mathrm{G}+$ \\
\hline
\end{tabular}

Laser characterization energy $=2.33 \mathrm{eV}$. The coefficient of determination $r^{2}=0.99$.

TABLE 5: Fitted parameters cathode energy $1.96 \mathrm{eV}(633 \mathrm{~nm})$.

\begin{tabular}{|c|c|c|c|c|c|c|}
\hline Peak & Type & Amplitude (a.u.) & Center $\left(\mathrm{cm}^{-1}\right)$ & FWHM $\left(\mathrm{cm}^{-1}\right)$ & $\%$ area & Interpretation \\
\hline 1 & Lorentz amp & 151.11 & 1323.10 & 93.29 & 22.77 & Nanotube defects \\
\hline 2 & Lorentz amp & 357.76 & 1333.53 & 57.58 & 22.91 & Amorphous carbon \\
\hline 3 & Lorentz amp & 339.74 & 1578.80 & 15.08 & 8.82 & G- \\
\hline 4 & Lorentz amp & 872.04 & 1582.78 & 26.85 & 39.53 & G0 \\
\hline 5 & Lorentz amp & 194.28 & 1617.77 & 18.10 & 5.90 & G+ \\
\hline
\end{tabular}

Laser characterization energy $=1.96 \mathrm{eV}$. The coefficient of determination $r^{2}=0.99$.

TABLE 6: Fitted parameters cathode energy $1.58 \mathrm{eV}(785 \mathrm{~nm})$.

\begin{tabular}{|c|c|c|c|c|c|c|}
\hline Peak & Type & Amplitude (a.u.) & Center $\left(\mathrm{cm}^{-1}\right)$ & FWHM $\left(\mathrm{cm}^{-1}\right)$ & $\%$ area & Interpretation \\
\hline 1 & Lorentz amp & 19.77 & 1269.26 & 205.75 & 22.77 & Amorphous carbon \\
\hline 2 & Lorentz amp & 148.16 & 1311.23 & 50.28 & 48.07 & Nanotube defects \\
\hline 3 & Lorentz amp & 51.43 & 1580.37 & 13.36 & 4.55 & $\mathrm{G}-$ \\
\hline 4 & Lorentz amp & 88.41 & 1584.86 & 37.35 & 20.97 & G0 \\
\hline 5 & Lorentz amp & 41.68 & 1614.55 & 15.19 & 4.14 & $\mathrm{G}+$ \\
\hline
\end{tabular}

Laser characterization energy $=1.58 \mathrm{eV}$. The coefficient of determination $r^{2}=0.98$. 
TABLE 7: Anode amorphous carbon concentration.

\begin{tabular}{lcc}
\hline Energy (ev) & Amorphous concentration (\%) & Defect index \\
\hline 2.33 & 61.67 & 1.24 \\
1.96 & 66.25 & 0.91 \\
1.5 & 56.72 & 0.49 \\
\hline
\end{tabular}

case correspond to carbon nanofoam. The widest signal, $1333.53 \mathrm{~cm}^{-1}$, corresponds to amorphous carbon. The last three signals correspond to tangential vibrations of the carbon atoms, the signal in $1578.80 \mathrm{~cm}^{-1}$ corresponds to the circumferential vibration, the second to the vibration in $1582.78 \mathrm{~cm}^{-1}$ corresponds to the carbon materials with $\mathrm{sp}^{2}$ hybridization, and the last signal in $1617.77 \mathrm{~cm}^{-1}$ corresponds to the vibration in the direction parallel to the axis tube.

Table 6 shows the adjustment of the G region or tangential vibrations; the first two signals correspond to defects in the nanotubes; of these, the narrowest signal, $1311.23 \mathrm{~cm}^{-1}$, corresponds to bent nanotubes that in this case correspond to carbon nanofoam. The widest signal, $1269.26 \mathrm{~cm}^{-1}$, corresponds to amorphous carbon. The last three signals correspond to tangential vibrations of the carbon atoms, the signal in $1580.37 \mathrm{~cm}^{-1}$ corresponds to the circumferential vibration, the second to the vibration in $1584.86 \mathrm{~cm}^{-1}$ corresponds to the carbon materials with $\mathrm{sp}^{2}$ hybridization, and the last signal in $1614.55 \mathrm{~cm}^{-1}$ corresponds to the vibration in the direction parallel to the axis tube.

Table 8 shows the proportions of amorphous carbon, and defects in nanostructures are summarized.

The physical processes that describe Raman and SEM characterizations are different, so even though the characterized area by SEM is larger, of about $25 \mu \mathrm{m}^{2}$ (for the analyzed micrography), the punctual excitation is smaller, approximately of $0.05 \mu \mathrm{m}^{2}$, while Raman characterization excited area is of less than $4 \mu \mathrm{m}^{2}$, obtaining punctual characterizations. Raman spectroscopy characterization is a resonant process; therefore, by exciting the sample with different lasers wavelength, diverse structures are excited; this depends on the electronic state densities of the samples.

The defects detected by Raman characterization do not correspond only to amorphous carbon; these defects correspond to topological defects, interstitial atoms, and vacancies. In the absence of a crystallographic structure corresponding to the amorphous carbon, a wider line shape is obtained, involving the mentioned defects, thus showing a more general view and reporting higher concentrations of amorphous carbon.

3.2.3. Transmission Electron Microscopy. The JEOL transmission electron microscope JEM-220Fs works with a field emission electron source (FEG) with $200 \mathrm{KeV}$ and a column energy filter (Omega Filter). The JEM-2200FS also uses a new rotation-free optical system to form the micrography, which facilitates the acquisition of TEM micrographies and diffraction patterns. It is possible to take high-contrast images, tomography, and work without the need of a dark room with the LCD camera. It has extensive processing tools of micrographies and computational analysis.

Figure 13 shows a multiwalled carbon nanotube with a catalytic mixture particle; in Figures 14 and 15, we can see the enlargement of the zones $\mathrm{z} 1$ and $\mathrm{z} 2$ and could measure the interplanar distance, which is close to the interplanar distance of the graphite.

The product deposited over the chamber walls and the grid structure is amorphous carbon. The product deposited over the electrode surfaces has similar structures in both with the difference that the structures deposited in the cathode are more free of amorphous carbon and catalyst particles than those deposited on the anode surface.

The discrepancy of the amorphous carbon concentrations localized on the cathode and anode is due to two factors, being these the heat exposure of the electrodes and the mass difference between the generated atoms cluster after the discharge. The heat exposure among the electrodes is different because of the anode discontinuities, also described as the catalytic mixture section, as shown in Figure 2(b). The anode is formed by eight teeth (catalytic mixture sections) and the cathode by only one point electrode; this implies that the temperature increase at the cathode is more frequent than the increment at one of the anode teeth. There is a formation of jet plasma after every discharge occurs; since the rotation of the anode base is counterclockwise, the plasma is dragged toward the cathode and covers it. This constant heat increases the mobility on the surface of the cathode, therefore allowing the atoms to be arranged on the areas of stable geometry or minimal energy.

When discharges occur, some materials are released from the anode; these can be a small set of atoms or a big set of atoms of catalytic mixture. The heavier elements cannot reach the cathode surface and are deposited on the surface of the teeth and in surrounding areas of the anode, that is, carbon nanofoam contaminated with amorphous carbon. Considering that on the cathode surface there are smaller and lighter set of atoms, their arrangement is more efficient, compared with the anode, where less frequent arrangement lapses occur and bigger and heavier molecules are located. Thus, the carbon nanofoam deposited on the cathode is less contaminated by amorphous carbon.

The electrical arc discharge is a complex and chaotic process. During this carbon nanofoam synthesis, most of the carbon nanostructures are produced, being impossible to produce $100 \%$ carbon nanofoam by this method. The modifications to this method increase the synthesis of carbon nanofoam; however, to increase the carbon nanofoam production rate, a more precise control of all the involved variables is required, that is, an automation of the system is needed.

\section{Conclusion}

The carbon product synthesized in the experiment was not deposited in spiderweb form. The product obtained was deposited over the electrode surfaces, chamber walls, and over the structure of the collecting grid in a black cotton way. 
TABLE 8: Cathode amorphous carbon concentration.

\begin{tabular}{lcc}
\hline Energy (ev) & Amorphous concentration (\%) & Defect index \\
\hline 2.33 & 19.78 & 4.62 \\
1.96 & 29.1 & 2.36 \\
1.5 & 22.77 & 0.84 \\
\hline
\end{tabular}

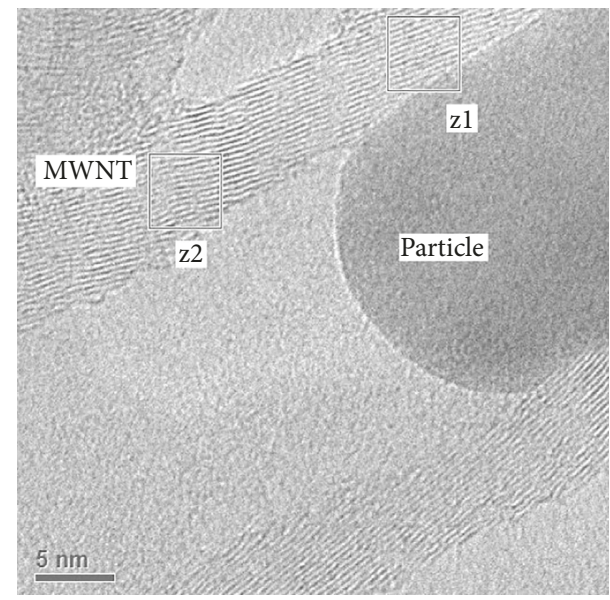

FIGURE 13: Multiwalled carbon nanotube.

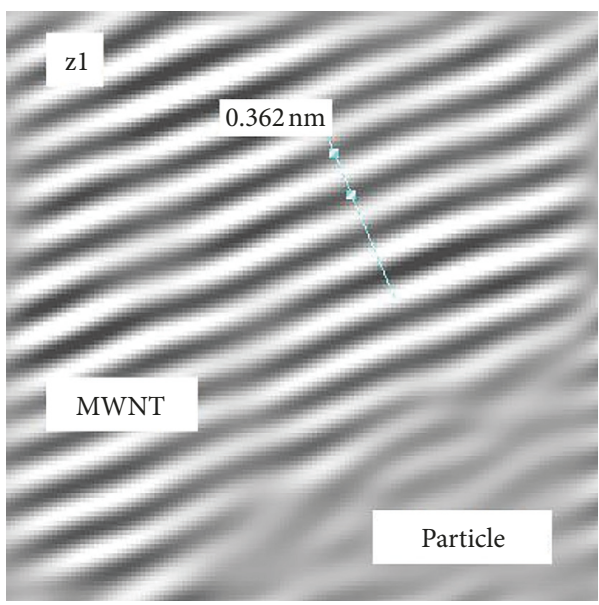

Figure 14: Zone z1 amplification.

In the characterization by scanning electron microscopy, it is found that the structures deposited over the anode surface have $35.66 \%$ amorphous carbon concentration, and the structures deposited over the cathode surface have $15.46 \%$ amorphous carbon concentration. The carbon nanofoam concentration over the anode surface is $11.94 \%$, and the carbon nanofoam concentration over the cathode surface is $81.82 \%$. The catalytic particle proportion on the anode is $52.4 \%$, and the catalytic particle proportion on the cathode is $2.71 \%$.

With the same technique, it is found that the carbon nanofoam structure is conformed by ropes with dimensions of 1 micron width and $100 \mathrm{~nm}$ length before breaking translation symmetry. The ropes are conformed by multiwalled carbon nanotubes, and the diameter is different in

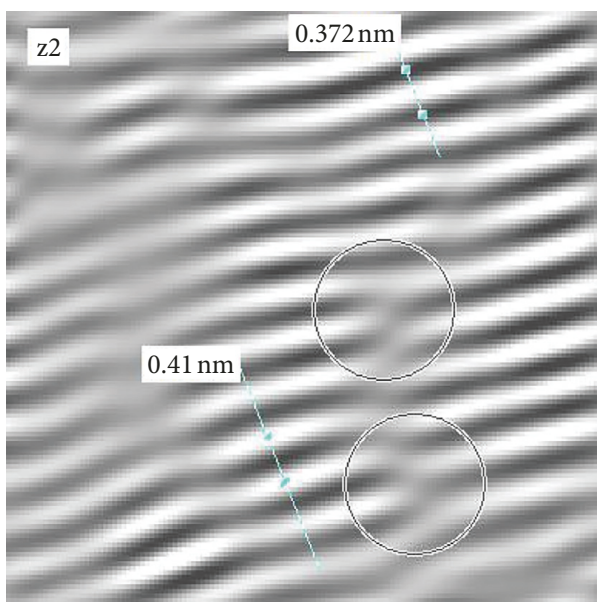

FIgURE 15: Zone z2 amplification.

anode and cathode, the mean diameter of the carbon nanotube at the anode is $30 \mathrm{~nm}$, and the mean diameter of the carbon nanotube at the cathode is $2.7 \mathrm{~nm}$.

With Raman spectroscopy characterization, different amorphous carbon concentrations were found because this depends on the laser excitation. With the $2.33 \mathrm{eV}$ laser energy, the proportion of amorphous carbon is $61.67 \%$; with the $1.96 \mathrm{eV}$ laser energy, the amorphous carbon proportion is $66.25 \%$; and with the $1.53 \mathrm{eV}$ laser energy, the amorphous carbon proportion is $56.72 \%$.

With these results, it can be seen that the growth and deposit on the electrodes are different for the anode (catalytic mixture section) and the cathode because the cathode pulse discharge frequency is greater. When the pulsed discharge occurs at the cathode, the catalytic mixture section is present; at the next discharge, the same cathode and other catalytic mixture sections are present. The time for the next discharge in the first catalytic mixture is the same time the anode makes a full lap; because of this, the temperature at the cathode is higher and constantly increasing. The different cathode and anode gap height affect the material deposit, only lighter material can attach to the cathode, and the heavy material attaches to the anode, like amorphous carbon and catalytic particles. This depends on the anode angular velocity too.

\section{Data Availability}

The data used to support the findings of this study are available from the corresponding author upon request.

\section{Conflicts of Interest}

The authors declare that there are no conflicts of interest regarding the publication of this paper.

\section{Acknowledgments}

The authors acknowledge the EDI grant by SIP/IPN. This research was partially supported by the projects 20181454, 20181441, and 20180472. 


\section{References}

[1] A. V. Rode, E. G. Gamaly, A. G. Christy et al., "Unconventional magnetism in all-carbon nanofoam," Physical Review B, vol. 70, no. 5, article 054407, 2004.

[2] N. Frese, S. Taylor Mitchell, C. Neumann, A. Bowers, A. Gölzhäuser, and K. Sattler, "Fundamental properties of high-quality carbon nanofoam: from low to high density," Beilstein Journal of Nanotechnology, vol. 7, pp. 2065-2073, 2016.

[3] K. O. Iwu, A. Lombardo, R. Sanz, S. Scirè, and S. Mirabella, "Facile synthesis of ni nanofoam for flexible and low-cost non-enzymatic glucose sensing," Sensors and Actuators B: Chemical, vol. 224, pp. 764-771, 2016.

[4] T. Iino and K. Nakamura, "Acoustic and acousto-optic characteristics of silicon nanofoam," Japanese Journal of Applied Physics, vol. 48, no. 7, article 07GE01, 2009.

[5] A. Kurek, R. Xalter, M. Stürzel, and R. Mülhaupt, "Silica nanofoam (nf) supported single-and dual-site catalysts for ethylene polymerization with morphology control and tailored bimodal molar mass distributions," Macromolecules, vol. 46, no. 23, pp. 9197-9201, 2013.

[6] L. Liu, I. Botos, Y. Wang et al., "Structural basis of toll-like receptor 3 signaling with double-stranded rna," Science, vol. 320, no. 5874, pp. 379-381, 2008.

[7] L. T. Johnston, M. M. Biener, J. C. Ye, T. F. Baumann, and S. O. Kucheyev, "Pore architecture of nanoporous gold and titania by hydrogen thermoporometry," Journal of Applied Physics, vol. 118, no. 2, article 025303, 2015.

[8] A. Zani, D. Dellasega, V. Russo, and M. Passoni, "Ultra-low density carbon foams produced by pulsed laser deposition," Carbon, vol. 56, pp. 358-365, 2013.

[9] S. T. Mitchell, N. Frese, A. Gölzhäuser, A. Bowers, and K. Sattler, "Ultralight carbon nanofoam from naphtalenemediated hydrothermal sucrose carbonization," Carbon, vol. 95, pp. 434-441, 2015.

[10] Y. B. Zel'dovich, Y. P. Raizer, W. D. Hayes, R. F. Probstein, and S. P. Gill, "Physics of shock waveshigh-temperature hydrodynamic phenomena, vol. 1," Journal of Applied Mechanics, vol. 34, no. 4, p. 1055, 1967.

[11] F. Neri, P. M. Ossi, and S. Trusso, "Propagation of laser generated plasmas through inert gases," Laser and Particle Beams, vol. 28, no. 1, pp. 53-59, 2010.

[12] M. Filipescu, P. M. Ossi, N. Santo, and M. Dinescu, "Radiofrequency assisted pulsed laser deposition of nanostructured wo x films," Applied Surface Science, vol. 255, no. 24, pp. 9699-9702, 2009.

[13] D. Saucedo-Jimenez, J. Ortiz-López, V. Garibay-Febles, and E. Palacios-González, "Sem and hrtem analysis of carbon nanostructures synthesized with a new pulsed electric arc discharge technique," Acta Microscopica, vol. 22, no. 3, pp. 289-299, 2013.

[14] A. A. Puretzky, H. Schittenhelm, X. Fan, M. J. Lance, L. F. Allard Jr., and D. B. Geohegan, "Investigations of singlewall carbon nanotube growth by time-restricted laser vaporization," Physical Review B, vol. 65, no. 24, article 245425, 2002.

[15] C. Liu, H. T. Cong, F. Li et al., "Semi-continuous synthesis of single-walled carbon nanotubes by a hydrogen arc discharge method," Carbon, vol. 37, no. 11, pp. 1865-1868, 1999.

[16] G. G. Tibbetts, C. A. Bernardo, D. W. Gorkiewicz, and R. L. Alig, "Role of sulfur in the production of carbon fibers in the vapor phase," Carbon, vol. 32, no. 4, pp. 569-576, 1994.
[17] M. S. Dresselhaus, G. Dresselhaus, R. Saito, and A. Jorio, "Raman spectroscopy of carbon nanotubes," Physics reports, vol. 409, no. 2, pp. 47-99, 2005.

[18] M. S. Dresselhaus, A. Jorio, A. G. Souza Filho, and R. Saito, "Defect characterization in graphene and carbon nanotubes using raman spectroscopy," Philosophical Transactions of the Royal Society of London A: Mathematical, Physical and Engineering Sciences, vol. 368, no. 1932, pp. 5355-5377, 2010.

[19] C. Andrea Ferrari, J. C. Meyer, V. Scardaci et al., "Raman spectrum of graphene and graphene layers," Physical Review Letters, vol. 97, no. 18, article 187401, 2006.

[20] S. Costa, E. Borowiak-Palen, M. Kruszynska, A. Bachmatiuk, and R. J. Kalenczuk, "Characterization of carbon nanotubes by raman spectroscopy," Materials Science-Poland, vol. 26, no. 2, pp. 433-441, 2008. 


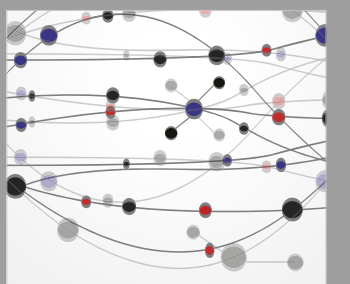

The Scientific World Journal
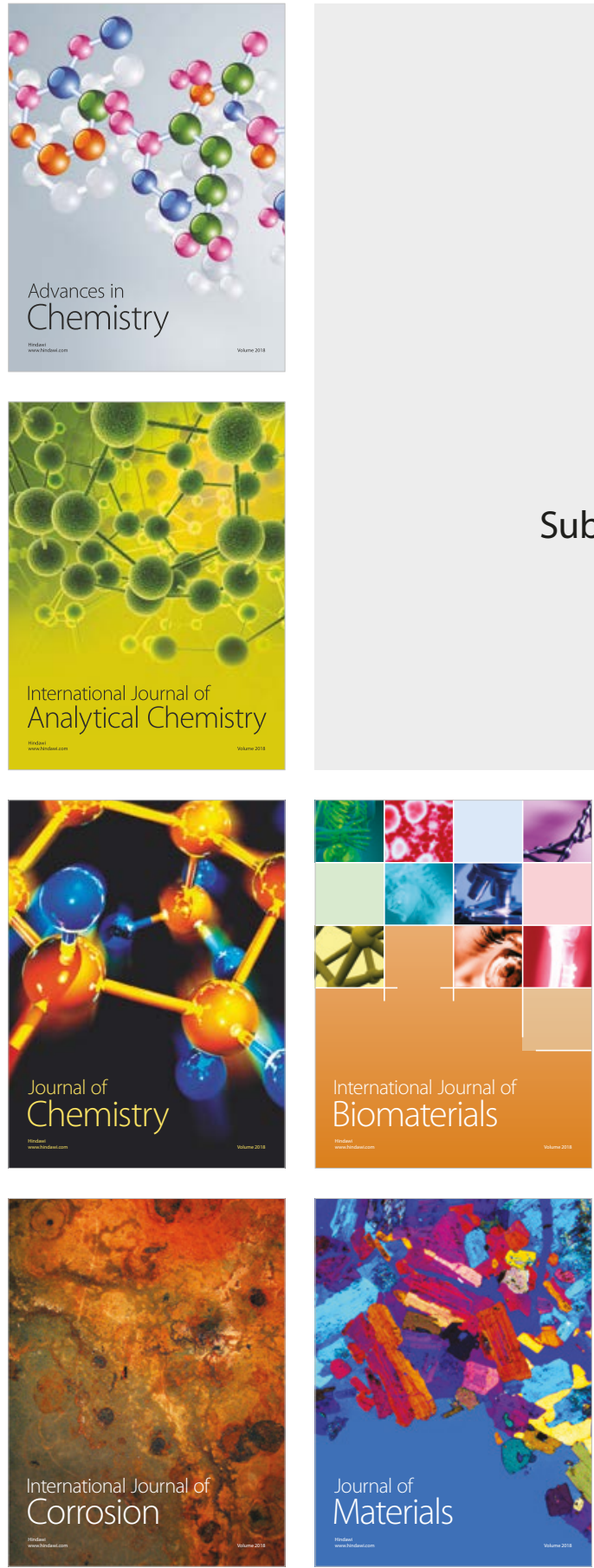

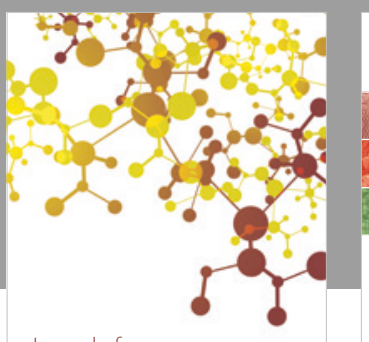

Journal of

Applied Chemistry
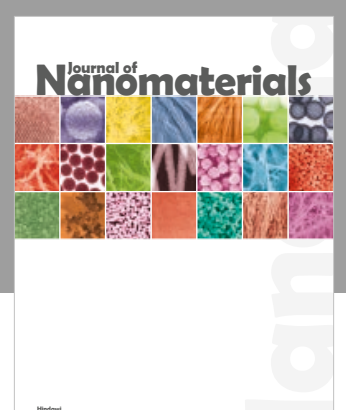

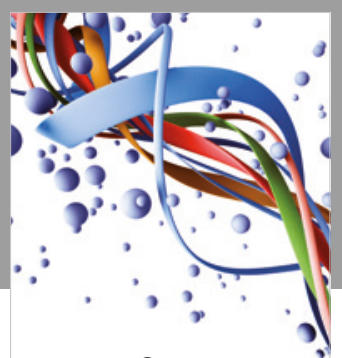

Scientifica

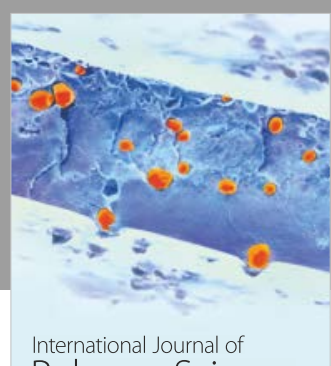

Polymer Science

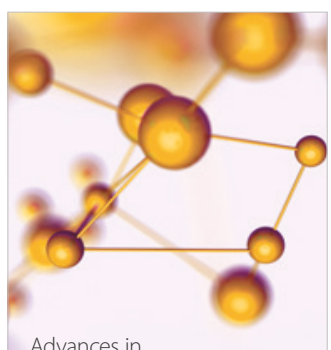

Physical Chemistry
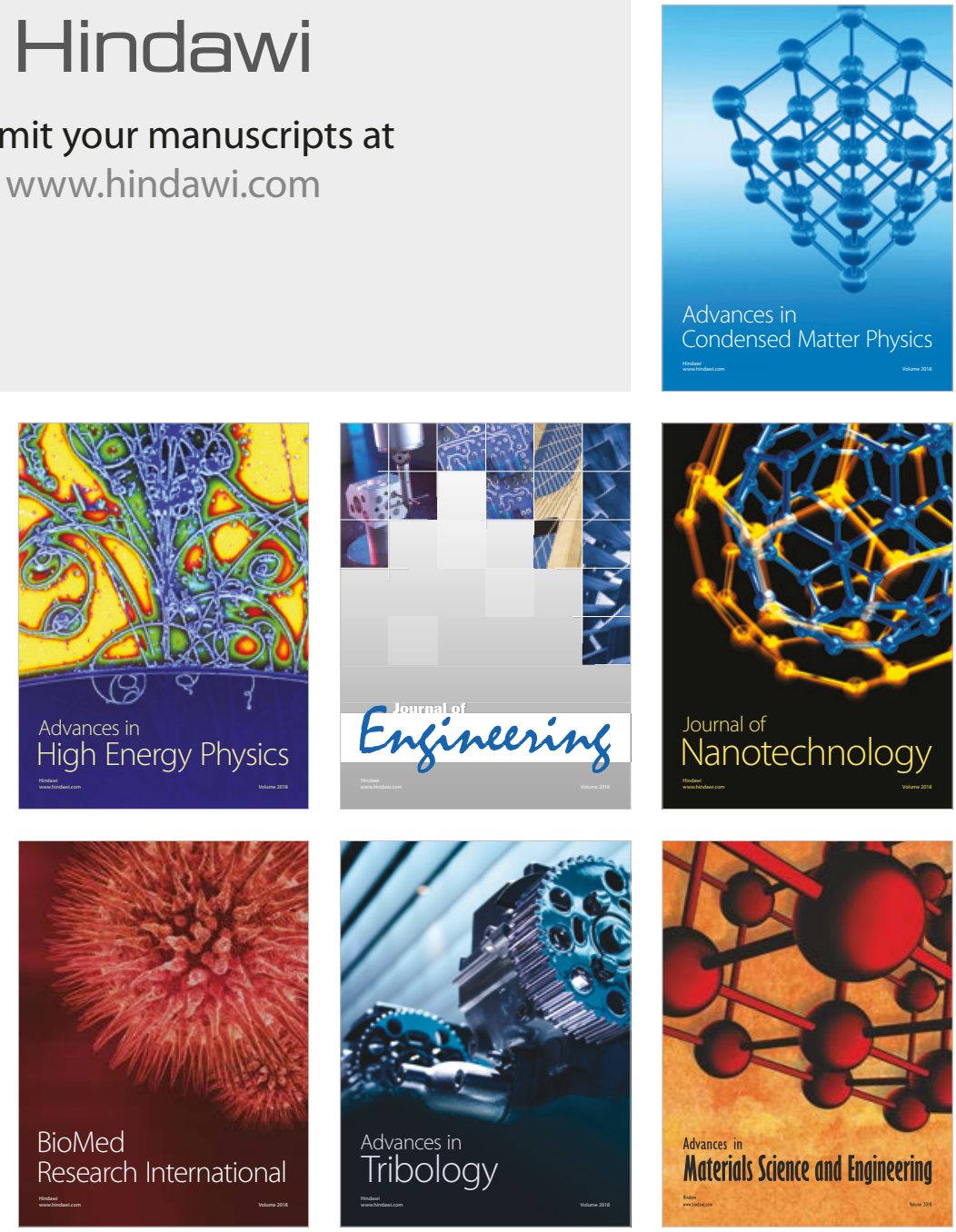